\title{
Holographic method for detecting amplitude and phase-shift errors and features in EUV ML reticle blanks
}

\author{
David Nijkerk, Norbert Koster, Eddy van Brug and Diederik Maas* \\ TNO Science and Industry, Stieltjesweg 1, 2628 CK Delft, The Netherlands
}

\begin{abstract}
A method for actinic inspection of EUV mask blanks is described, in which EUV photoresist is applied to the blank, flood exposed with EUV, and developed. The effect of both phase and reflectivity defects on the reticle is described in terms of a variation in intensity and phase of the standing wave in the resist. Thin film simulations are performed to evaluate the contrast generating mechanism for various blank defects. The method was introduced earlier by others ${ }^{3}$ and was shown in experiments to transfer reflectivity defects on the reticle to the developed resist. We propose to reevaluate the technique with current state-of-the-industry capabilities of resist processing, contamination control and inspection. Various possible development directions are described.
\end{abstract}

Keywords: EUV, defect detection, interference lithography, reticle inspection, blank inspection

\section{INTRODUCTION}

Extreme ultraviolet lithography (EUVL) relies on the use of almost defect free blanks consisting of multi-layers (ML) on a thick mirror substrate. The defect density must be below 0.003 defects $/ \mathrm{cm}^{2}$. Typical defects could be a local phase shift defect resulting from a bilayer thickness mismatch or a buried particle, both occurring at an arbitrary depth of the ML stack. Not only the fabrication of these reticle blanks, but also qualification of the blanks with regards to absorbing and/or phase shift defects and/or features in the Multi-Layer (ML) on the thick mirror substrate remains a key challenge for the implementation of EUVL ${ }^{1}$. In this paper we propose an alternate method of detecting phase and amplitude variations resulting from errors and features in and on the ML stack.

During wafer exposure, the electric field emanating from the reticle is projected onto the wafer. Any features that are printed in the resist on the wafer must originate from contrast features in this projected electric field. The basis of the current method is that the contrast in the electric field at the wafer, in as far as they originate from errors and features on the mask rather than from imperfections in the projection optics, must be present in the electric field at the mask as well. A reticle inspection technique like $\mathrm{AIMS}^{2}$ makes use of this fact by projecting the field at the reticle with a large magnification onto a EUV sensitive spatially resolved detector.

The idea of the current method is to record the electric field at the mask directly and consequently capture precisely those features and errors that will ultimately print on the wafer. The electric field at the faulty reticle or blank is captured by printing its image in photoresist by means of a holographic method making use of interference of incident and reflected beam. This method was described some years ago by other authors ${ }^{3,4,5}$ yet we feel it is worth bringing it to the attention of the industry again, as no cost-effective mask blank inspection solution in the mask infrastructure is yet developed ${ }^{6}$.

In Section 2, the present method will be explained in more detail. In Section 3 we will present the results from simulations of the method. Section 4 will dwell upon the next steps to be taken towards validating the technique and assessing its feasibility for industrial application. Lastly, conclusions will be drawn in Section 5.

*Corresponding author: Diederik.Maas@tno.nl; phone+31 15259 2368; www.tno.nl

Extreme Ultraviolet (EUV) Lithography, edited by Bruno M. La Fontaine, Proc. of SPIE Vol. 7636, $763622 \cdot$ ㅇ 2010 SPIE · CCC code: 0277-786X/10/\$18 · doi: 10.1117/12.846505 


\section{OUTLINE OF THE TECHNIQUE}

First, consider the life of the electric field as it propagates through the optical system in a wafer stepper. A homogeneous field is directed from an illuminator system towards the reticle. On the way to the wafer, variations in phase and amplitude are introduced. Under the assumption of error free imaging, these variations result from features at the reticle. These features may be intentional, e.g. caused by absorber or phase shifting structures, or unintentional, caused by local attenuation or phase defects in the reticle. Regardless of the cause, these features are present in the field that propagates from reticle level to wafer level.

In a wafer stepper the features in the electric field result in inhomogeneities in the intensity profile at wafer level through constructive and destructive interference of the diffraction orders that pass through the optical system. Via the same mechanism of interference, these features result in inhomogeneities at reticle level through combination of the homogeneous incident beam that originates from the illuminator and the beam that is reflected from the reticle. Capturing this interference pattern at the reticle, we effectively use the illuminator beam as a reference beam in a holographic recording of the electric field emanating from the reticle. In the present defect detection method, it is this interference pattern that is utilized to flag defects that would ultimately print on the wafer, if the reticle were used in a wafer stepper.

The interference pattern is captured in a standing wave in a EUV sensitive photoresist layer on the reticle. This is akin to the standing wave effect that occurs in the resist layer on a wafer. The standing wave causes a variation in dissolution rate over the depth of the resist layer. In DUV photolithography on the wafer, the standing wave effect is an undesired side effect that brings about swing curve and side wall ridges and must be reduced carefully by using index matching antireflective coatings and post exposure bakes and so on. In contrast, in the present defect detection method, the trick is to play around with clearing dose, thickness, sensitivity and processing parameters of the photoresist to make defects rather show up in the developed resist. The standing wave in the resist varies in phase with varying reticle blank features. This phase difference of the standing wave must be detected as contrast in the developed resist layer.

In Figure 1, the method is sketched qualitatively. On the left, the incoming and reflected radiation are represented with solid arrows and dashed arrows, respectively. In the figure, the amplitudes of the electric field near the surface of the EUV reflector are indicated with the solid and dashed waves. The intensity of the combined wave is represented with the dotted line. On the right the reflected wave has experienced a phase shift with respect to the situation on the left, with a resulting phase change of the combined intensity.
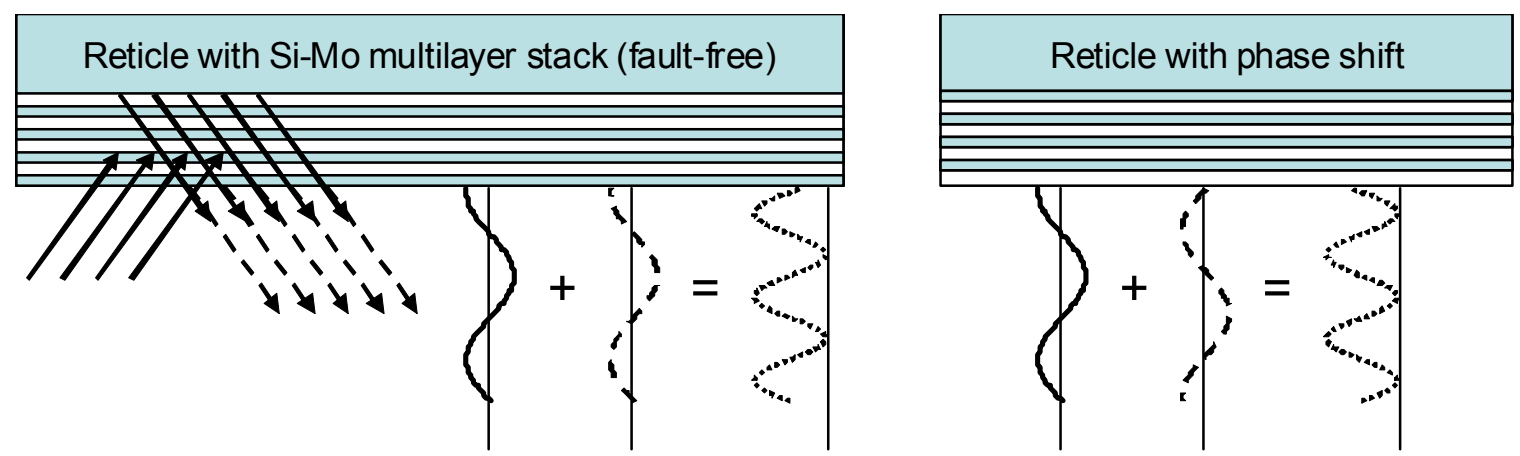

Figure 1. Pictorial example of interference pattern for defect free reticle (left) and reticle with phase shift defect (right).

The intensity profile over the depth of the resist is given by the common expression of the superposition of two interfering waves $\mathbf{E}_{\text {ill }}$ and $\mathbf{E}_{\text {refl }}$ for the illuminating and reflected wave respectively, which, when rewritten in terms of the intensities of the waves, yields ${ }^{7}$ 


$$
I=I_{\text {ill }}+I_{\text {refl }}+\sqrt{2 I_{\text {ill }} I_{\text {refl }}} \cos (\delta+\varepsilon) .
$$

The phase constant $\delta$ depends on both the depth in the resist and the angle between $\mathbf{E}_{\text {ill }}$ and $\mathbf{E}_{\text {refl }}$ and is approximately $4 \pi z / \lambda$, and $\varepsilon$ is an unknown phase offset that depends on the state of the inspected multi-layer and resist stack. Thus the information to be retrieved is contained in the intensity of the reflected wave $I_{\text {refl }}$ and on the phase constant $\varepsilon$. Both $I_{\text {refl }}$ and $\varepsilon$ are functions of $\mathrm{x}$ and $\mathrm{y}$, the positions on the reticle. Thus, the resist layer is considered as an array of little resist 'pillars', each pillar containing the information of its particular location on the reticle. This is a rather simplified view as in practice pillar-to-pillar cross-over of the intensities will take place, but it helps in describing the technique.

The explicit mathematical description of the contrast mechanism tentatively provokes the consideration of two modes of operation. When probing the resist over a depth $\Delta z=\lambda / 2$, the influence of the oscillatory cosine term will vanish (assuming that the intensity can be averaged over depth) so that the contrast is given by the second term, $I_{\text {refl }}$ and the influence of the phase offset $\varepsilon$ drops out of the equation for all resist pillars. Alternatively, when probing the resist at the top, the contrast is also given by the third term, $\sqrt{ } 2 I_{\mathrm{ill}} \cdot I_{\text {refl }} \cdot \cos (\delta+\varepsilon)$. The third term provides contrast even when $I_{\text {refl }}$ maintains a constant value over the reticle. Thus, the former mode (which we will call 'amplitude mode') is more susceptible to reflection errors in the reticle, whereas the latter mode ('phase mode') is more susceptible to phase errors.

Since the period of the standing wave is on the order of half the illuminating wavelength in the resist, the resolving capabilities of the resist must be very high. The method then could benefit from the capability to resolve topographic differences of only a few nm, notably in phase mode. This is not trivial with standard optical techniques especially at sufficiently high lateral resolution but it can be done, e.g. using ellipsometry, interferometry, confocal microscopy, et cetera. In any case it is not essential to the technique. Another mechanism that could be used to distinguish among defect types is the variation in slope of the resist image, especially when the standing wave can be resolved.

The contrast is increased considerably when the entire resist pillar remains standing or is removed during development, depending on $\mathrm{I}_{\text {refl }}$ and $\varepsilon$. This could be achieved when the intensity in the resist is averaged, and when using thin resist layers, e.g. on the order of the period of the standing wave.

Amplitude mode
dissolution change
averaged in entire
layer
$\begin{gathered}\text { Phase mode: dissolution change } \\
\text { at the top surface }\end{gathered}$
\begin{tabular}{|c|c|}
\hline Amplitude mode & Phase mode \\
\hline $\begin{array}{c}\text { sensitive to amplitude } \\
\text { variation }\end{array}$ & $\begin{array}{c}\text { sensitive to amplitude } \\
\text { and phase variation }\end{array}$ \\
\hline $\begin{array}{c}\text { Thin resist layer } \\
\text { beneficial }\end{array}$ & $\begin{array}{c}\text { Inspection with high } \\
\text { depth resolution } \\
\text { beneficial }\end{array}$ \\
\hline Low resolution resist & High resolution resist \\
\hline
\end{tabular}

Figure 2. Two modes of operation: contrast based on averaged intensity in the full resist layer or at top of the resist layer. 
The locations of the features in the developed resist thus detected with existing infrastructure are stored in a data base. After clearing the blank of remaining developed resist, the next step could be to repair the defect, followed by a check whether the repairing and/or cleaning step was successful.

For the technique, we coin the acronym EDICT, for

E Exposure of the resist on the multilayer on the reticle blank

D Development of the exposed resist

I Inspection of the developed pattern

C Cleaning of the reticle to remove all traces of the resist

$\mathrm{T} \quad$ Test whether the reticle is sufficiently cleaned

The 'reticle blank' mentioned in the Exposure step may be read as generic optical element with multilayer designed for the actinic light. It should be realized that the technique is applicable to multilayer mirror inspection as well. Inspection of the developed pattern could ideally be performed with existing (DUV and/or EUV) reticle inspection or wafer inspection infrastructure, but a dedicated inspection tool may also be envisaged. Implementation of the $\underline{\text { Cleaning step }}$

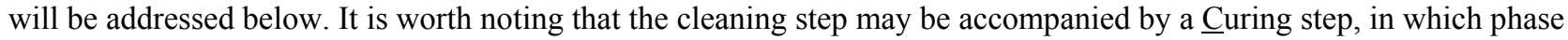
errors are repaired with local modification methods such as e.g. charged particle beam exposure.

We want to stress that more than a fundamental difference in operation, the distinction between 'phase mode' and 'amplitude mode' is a theoretical tool to provide insight in the contrast generating mechanisms, both optically and lithographically. The true contrast in practice will be the result of a balance between amplitude errors, phase errors and resist processing and illumination parameters.

\section{SIMULATION OF BLANK ERRORS}

The feasibility of the method can be assessed initially using computer simulations of the underlying illumination process. A thin film simulation software tool (MacLeod by Thin Film Center) has been used to determine the phase and reflectivity changes when an anomaly is present in a multilayer stack.

A multilayer stack consisting of 60 Molybdenum Silicon bilayers was defined, having a peak reflectivity of $75 \%$ (theoretical value) at $13.5 \mathrm{~nm}$ radiation and $6^{\circ}$ angle of incidence. When coating the stack with a layer of resist, the reflectivity drops due to absorption in the resist. The complex refractive index of resist used as input in the thin film model is $n=1-0.0242+0.0054 \cdot \mathrm{i}$. This is the measured value for PMMA ${ }^{8}$, which is considered here to be representative for any typical organic photoresist. The reflectivity spectrum is show in Figure 3.

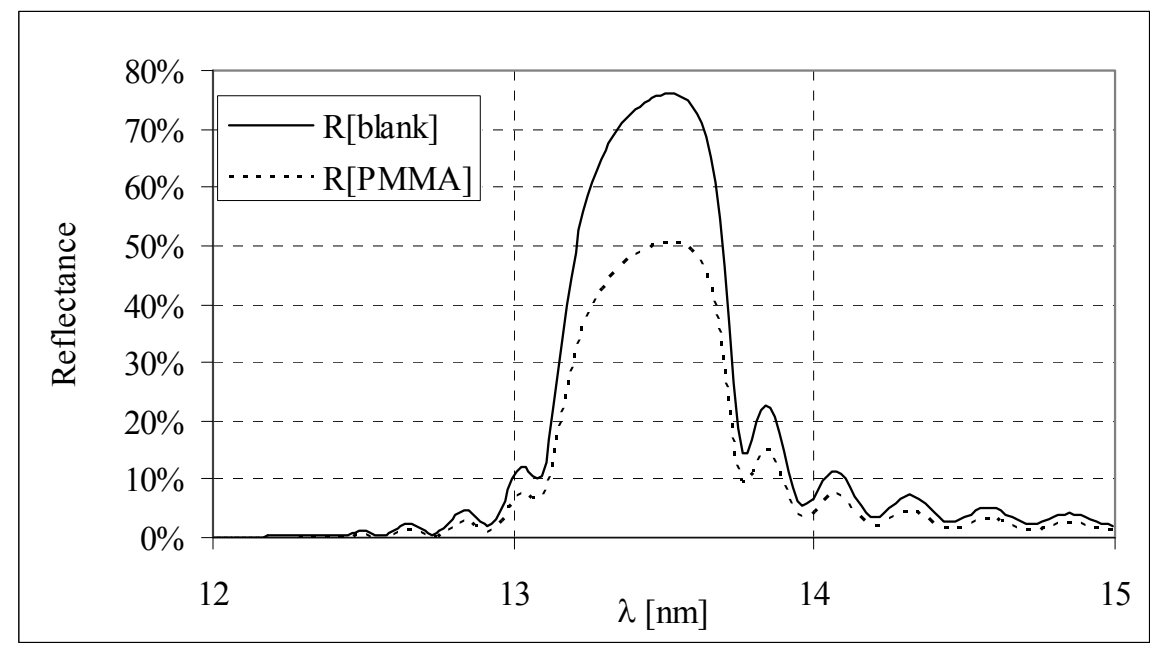

Figure 3. Reflectivity of multilayer stack with and without $40 \mathrm{~nm}$ PMMA 
An interesting quantity to monitor is the electric field inside the multilayer stack. Figure 4 shows the electric field in $\mathrm{V} / \mathrm{m}$ as a function of depth in the layer stack with $40 \mathrm{~nm}$ PMMA. The units on the $x$-axis are full wave optical thickness (FWOT), i.e. a physical thickness corresponding to the vacuum wavelength $(13.5 \mathrm{~nm})$ divided by the real part of the refractive index. The graph shows the oscillatory behaviour typical of a standing wave, and in addition an envelope that remains constant in the resist layer and decreases with depth in the multilayer stack. The decreasing electric field in the multilayer gives an indication of the extent to which a fault in the multilayer affects the reflected field. The electric field drops to $5 \%$ at $20 \mathrm{FWOT}$, thus faults below 20 FWOT (which corresponds to approximately 34 bilayers below the top surface) contribute $5 \%$ or less to the reflected field relative to the contribution at the top of the stack. This has the consequence that a fault inside the stack may be acceptable if it is buried deep enough.

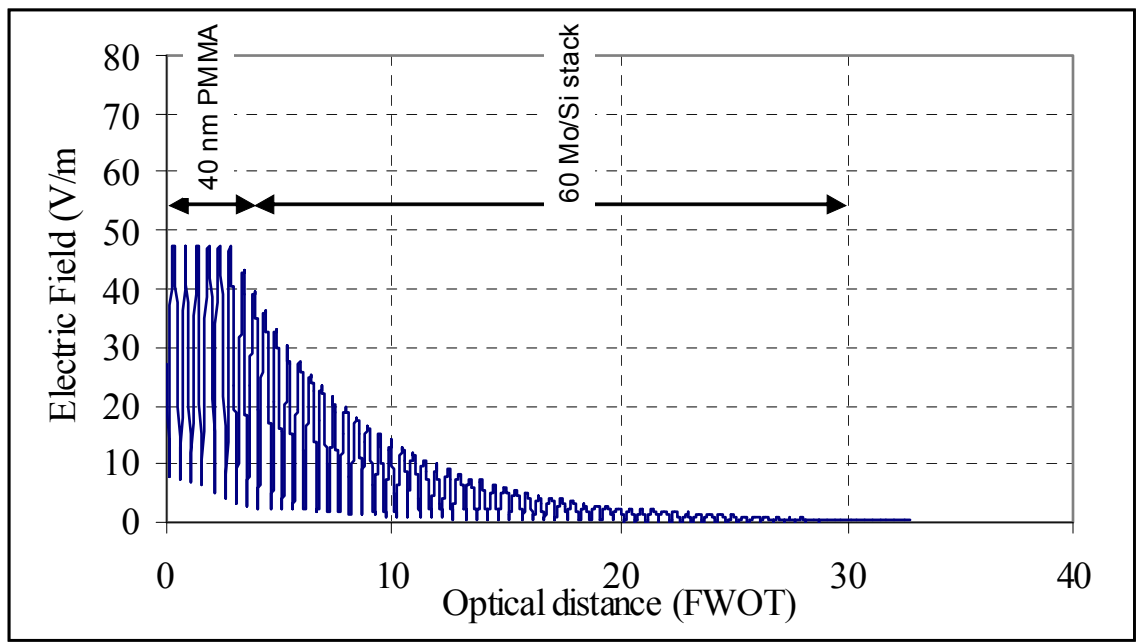

Figure 4. Electric field in layer stack with $40 \mathrm{~nm}$ PMMA

The shape of the standing wave in the resist layer is affected by the underlying multilayer stack. For two cases deviating from a nominal stack, the electric field was computed in a thin film simulation. One case relates to a reduced thickness of the of the Mo layer in the fourth bilayer by $50 \%$. The other relates to a buried silver layer below the third bilayer. The results are shown in Figure 5. Note that due to the nature of the 1D thin film simulation, these errors extend throughout the entire individual layer. In practice the errors will likely be confined laterally so that the effect is somewhat different than plotted below. 


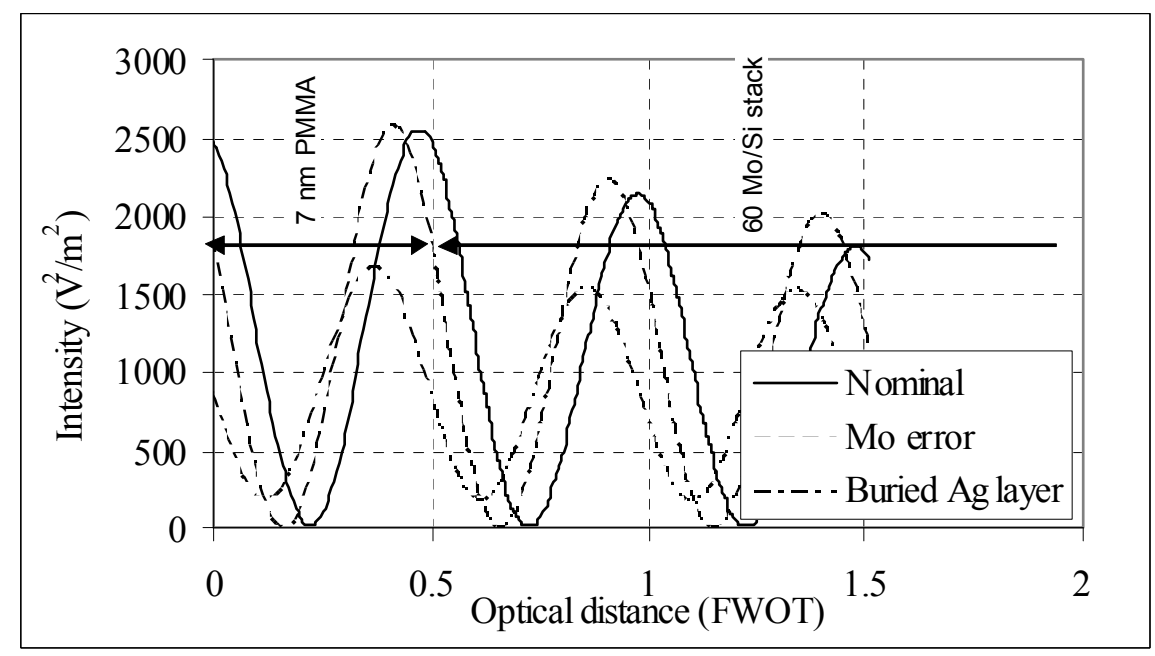

Figure 5. Intensity of the optical field in layer stack with errors

Now suppose that the reticle is covered with a positive tone high resolution resist. Looking at the intensity levels in the examples above, it is seen that when the resist clearing dose corresponds to an intensity level of $1700 \mathrm{~V}^{2} / \mathrm{m}^{2}$, the resist over a buried silver layer is developed entirely, the resist over a multilayer with a molybdenum error is developed up to 0.3 FWOT $(\approx 4 \mathrm{~nm})$, whereas the resist over the nominal multilayer is not developed. The silver layer is thus detected due to the difference in reflected intensity, whereas the molybdenum error is detected due to the difference in reflected phase.

The resist must have sufficient resolution capability to distinguish between the nominal stack and the stack with phase (Mo) error, as blurring the intensities by more than a few nm will make it impossible to differentiate between the intensity curves. On the other hand, when the intensity profile is blurred throughout the resist layer thickness, the contrast arises from the average intensity level. In that case, the nominal stack and the Mo error stack cannot be distinguished, but the buried silver layer is still distinguished, because the reflected intensity of the latter stack is different. In the underlying situations, the contrast is described mainly by the second term of Equation (1) (i.e. arising from a difference in reflectivity) or the third term (i.e. arising from a difference in phase).

\section{EXPERIMENTAL VERIFICATION}

\subsection{Experimental results in literature}

As mentioned earlier, the method was introduced by Spector et al. ${ }^{3}$. These authors performed initial experimental validation of the method. An EUV mask blank with programmed defects was coated with resist, flood exposed using synchrotron radiation and subsequently partially developed. The programmed defects were created in the multilayer coating using a focused ion beam. Implantation of $\mathrm{Ga}^{+}$resulted in local reflectivity loss depending on the ion dose. It was shown that the method works, in the sense that bumps or pits of resist remain after development, marking sites with amplitude defects. The resist parameters could be tuned to increase the size of the bumps and pits and consequently the sensitivity of the technique. Assessment of the sensitivity using standard inspection machines was performed by creating resist bumps using e-beam lithography and scanning with an unpatterned wafer inspection tool (Surfscan SP1), in order to verify whether the defect sites can be located adequately using optical inspection techniques, in manual or automatic mode. The size of the programmed defects was typically $10 \mu \mathrm{m}$ square. Defects considerably smaller than $10 \mu \mathrm{m}$ square were not visible due to contamination in the resist. The changes in resist height depend not only on the particular defect, but also on the thickness of the applied resist film. 
An alternative method was described as well, in which resist was applied to a transparent membrane placed in close proximity to the reticle, rather than to the reticle itself. In this case, as the light propagates away from the mask, phase defects cause additional interference variation, making them easier to detect than with the direct method. Difficulties with handling a transparent membrane the size of an entire mask blank aside, this method has the additional advantage that one possible source of contamination of the reticle is eliminated.

\subsection{Outlook for further technology assessment}

We propose to supplement the experimental verification of the technique by looking at a number of other aspects. As we have shown in the previous section, phase information is contained in the phase of the standing wave intensity profile. In the previous analysis by Spector et al. it was assumed that the standing wave in the resist can be ignored. This is certainly the case when the standing wave cannot be resolved due to limitations in the resist. However, the capabilities of resist, and the capabilities of applying ultra thin layers of resist, have progressed and it is worth reconsidering the standing wave effect as a possibility to detect phase errors. This would circumvent the need for a membrane (i.e. the alternative method described in section 4.1). To obtain insight in the limitations in resists exposed with EUV an elaborate simulator has been developed ${ }^{9}$ that could supplement experiments. As far as the function of a membrane as protection from contamination is concerned, we are confident that this is not required. We have obtained excellent results with multilayer cleaning, showing that a multilayer mirror can be cleaned without damage using a hydrogen plasma ${ }^{10}$.

One important aspect of the present technique is the influence of contamination on the detection efficiency and detection limit. In recent years a lot of effort has been put into mitigating the influence of contamination in EUV systems. Although it remains difficult to control, it has been shown that the ASML Alpha Demo Tool has the issue of contamination well under control ${ }^{11}$. It is expected that the performance limit with regards to lateral resolution, in the previous study imposed by resist contamination, has shifted considerably.

A possible topic to extend the study is looking at the merits of using ultra thin resist layers. The previous study used resist thicknesses of $80-100 \mathrm{~nm}$. It has been shown that e.g. PMMA forms nice films of even smaller thickness ${ }^{12}$. In these proceedings ${ }^{13}$ it is shown that even thinner layers of $5 \mathrm{~nm}$ of HSQ resist can be applied without difficulty.

In section 2, we listed several optical techniques intrinsically capable of resolving $\mathrm{nm}$ thickness variations on a substrate. One other inspection method that is easily capable of detecting topographic features on the order of several nm is charged particle beam microscopy (SEM, HIM). A nice demonstration of the capabilities of HIM (Helium Ion Microscopy) is shown in these proceedings ${ }^{13}$. A logical step in the technology assessment would be to determine the detection efficiency of the defects on the reticle with either of these techniques. The balance between defect size, or more appropriately, size of the feature in the resist on the one hand, and resolving capabilities of the surface analysis technique on the other, is an important aspect in assessing the sensitivity, and consequently, speed of the inspection method. This aspect of throughput, determined by signal-to-noise, defect size and resolution, has been addressed in a previous publication ${ }^{14}$. In that paper, a novel wafer inspection technique using multiple low resolution electron beams is described. A throughput model was developed that can easily be adapted for blank inspection with an arbitrary surface analysis technique.

The previous study used synchrotron radiation for the resist exposure. It is well worth noting that this is not a necessity. The coherence and chromaticity requirements of the illumination are relaxed and most industrial and current laboratory EUV sources will suffice for this technique.

\section{CONCLUSION}

This paper describes a technique for at-wavelength mask blank inspection that can be implemented fast and at relatively little technological effort. In this technique photoresist is applied to a mask blank, exposed with EUV and developed. Phase and reflectivity defects in the blank are then transferred to the developed resist. Initial experimental studies performed in the past by others ${ }^{5}$ have shown that the technique is viable. We feel it is worthwhile to bring the technique to the notice of the industry once more in the absence of a mature EUV mask blank inspection platform. 
The current publication takes a complementary view on the contrast generating mechanism of the technique. It thus suggests a direct approach to detect phase defects as well as reflectivity defects.

One of the strengths of this technique is its relying on existing industry infrastructure. The consequence is that only little development effort is expected. Having relevant equipment, network and experience to initiate further evaluation of the inspection method, we propose to reevaluate the technique with the current state-of-the-industry capabilities with respect to resist processing, contamination control and inspection. The previous experimental results looked promising. Next objectives should be on the areas of pushing down the detection limit (lateral resolution, phase error, reflectivity error), assessing inspection speed, and evaluating the technological implementation. The matter of detection capabilities should also be linked to whether a defect will actually print on a wafer.

\section{REFERENCES}

[1] Peters, J. H., Tonk, C., Spriegel, D., Han, H. S., Cho, W. and Wurm, S., “EUV blank inspection,” Proc. SPIE 6792, 67920F (2008).

[2] Bechstein, K. H., Esselbach, M., Harnisch, W., Rosenkranz, N., Scherübl, T., Seitz, H., Windpassinger, R., Zibold, A., Kuerz, P. and Sohmen, E., "EUV AIMS @ Zeiss - AIMSTM EUV Development Design Study," Sematech EUVL Symposium 2003, http://www.sematech.org/meetings/archives/litho/euvl/20030930/posters/Poster 047 - Bechste.pdf

[3] Spector, S. J., White, D. L., Tennant, D. M., Luo, P. and Wood II, O. R., "Rapid at-wavelength inspection of EUV mask blanks by photoresist transfer," Proc. SPIE 3546, p. 548 (1998).

[4] Spector, S. J., Luo, P., Novembre, A. E., Ocola, L. E., White, D. L., Tennant, D. M. and Wood II, O. R., "Development of a technique for rapid at-wavelength inspection of EUV mask blanks," Proc. SPIE 3676, p. 606 (1999).

[5] Spector, S. J., White, D. L., Tennant, D. M., Ocola, L. E., Novembre, A. E., Peabody, M. L. and Wood II, O. R., "Technique for rapid at-wavelength inspection of extreme ultraviolet mask blanks," J. Vac. Sci. Technol. B 17 p. 3003 (1999).

[6] ITRS Lithography, p. 5, www.itrs.net (2009).

[7] E. Hecht, [Optics], Addison Wesley Longman, Reading, p. 378-379 (1998).

[8] Kwark, Y. J., Bravo-Vasquez, J. P., Chandhok, M., Cao, H., Deng, H. and Gullikson, E., "Absorbance measurement of polymers at extreme ultraviolet wavelength: Correlation between experimental and theoretical calculations," J. Vac. Sci. Technol. B Volume 24, Issue 4, pp. 1822-1826 (2006).

[9] Drygiannakis, D., Nijkerk, M. D., Patsis, G. P., Kokkoris, G., Raptis, I., Leunissen, L. H. A. and Gogolides, E., "Simulation of the combined effects of polymer size, acid diffusion length, and EUV secondary electron blur on resist line-edge roughness," Proc. SPIE 6519, 65193T (2007).

${ }^{[10]}$ Koster, N. B., Koops, R., Agovic, K., de Groote, F. P. J., Wieringa, F. P. and Meijerink, M. G. H., "Shielded plasmas for cleaning EUV mirrors," Sematech EUVL Symposium 2008, http://www.sematech.org/meetings/archives/litho/8285/pres/CP-03-Koster.pdf

[11] Okoroanyanwu, U., Jiang, A., Dittmar, K., Fahr, T., Laursen, T., Wood II, O. R., Cummings, K. D., Holfeld, C., Bubke, K., Peters, J. and La Fontaine, B. M., "Monitoring EUV reticle molecular contamination on ASML's alpha demo tool," these proceedings, SPIE 7636-16 (2010).

[12] Kokkinis, A., Valamontes, E. S. and Raptis, I., "Dissolution properties of ultrathin photoresist films with multiwavelength interferometry," J. Phys.: Conference Series 10 p. 401-404 (2005).

[13] Maas, D.J., van Veldhoven, E., Chen, P., Sidorkin, V., Salemink, H.W.M., van der Drift, E., and Alkemade, P.F.A. "Nanofabrication with a Helium Ion Microscope," these proceedings, SPIE 7638-143 (2010).

[14] van Himbergen, H. M. P., Nijkerk, M. D., de Jager, P. W. H., Hosman, T. C. and Kruit, P., "High throughput defect detection with multiple parallel electron beams," J. Vac. Sci. Technol. B25(6), pp. 2521-2525 (2007). 